\title{
SYMMETRY REDUCTION OF ASYMMETRIC HEAVENLY EQUATION AND 2+1-DIMENSIONAL BI-HAMILTONIAN SYSTEM
}

\author{
DEVRIM YAZICI AND HAKAN SERT
}

Communicated by Alexandar B. Yanovski

\begin{abstract}
Asymmetric heavenly equation, presented in a two-component form, is known to be 3+1-dimensional bi-Hamiltonian system. We show that symmetry reduction of this equation yields a new two component 2+1-dimensional integrable bi-Hamiltonian system. We prove that this new 2+1-dimensional system admits bi-Hamiltonian structure, so that it is integrable according to Magri's theorem.
\end{abstract}

\section{Introduction}

Asymmetric heavenly equation was obtained as one of the canonical equations in the classification of nonlinear second order partial differential equations that possess partner symmetries [1]. The asymmetric heavenly equation in 3+1-dimension is given by

$$
u_{t x} u_{t y}-u_{t t} u_{x y}+a u_{t z}+b u_{x z}+c u_{x x}=0
$$

where $u$ is the unknown function that depends on the four independent variables $t, x, y, z$ and the subscripts denote partial derivatives of $u$, e.g., $u_{t x}=\partial^{2} u / \partial t \partial x$, $u_{x x}=\partial^{2} u / \partial x^{2} \ldots$, while $a, b, c$ are constants. By choosing $u_{t}=v$ as the second unknown, we have converted the asymmetric heavenly equation to the twocomponent evolution system [2]

$$
u_{t}=v, \quad v_{t}=\frac{1}{u_{x y}}\left(v_{x} v_{y}+a v_{z}+b u_{x z}+c u_{x x}\right) \equiv Q .
$$

The physical significance of the singe scalar equation (1) follows from the fact that it is equivalent to complex Einstein field equations for (anti-)self-dual gravitational fields [3], with $u$ being the metric potential.

In [2] we found all point symmetries of asymmetric heavenly equation. In general, a symmetry reduction of this equation has no Hamiltonian structure. Here we show that one particular symmetry reduction yields a two-component 2+1-dimensional new bi-Hamiltonian integrable system. For this system we present Hamiltonian 
structure, recursion operator, Lax pair, Lie point symmetries and integrals of motion.

In Section 2, we present new 2+1-dimensional integrable system in two-component form obtained by a symmetry reduction of asymmetric heavenly system.

In Section 3, we obtain the first Hamiltonian structure of this system of equations. We start with a degenerate Lagrangian and construct its Dirac bracket [4] to find a Hamiltonian operator.

In Section 4, we construct a recursion operator in a matrix form using the results presented in [2]. The recursion operator and operator of the symmetry condition form a Lax pair of the Olver-Ibragimov-Shabat type for the two-component system [5].

In Section 5, we give explicitly the second Hamiltonian structure which shows that the asymmetric heavenly equation is an integrable bi-Hamiltonian system.

In Section 6, we present all point symmetries of the reduced system. Using the inverse Noether theorem for Hamiltonian symmetries, we determine the corresponding integrals of motion.

\section{Symmetry Reduction of Asymmetric Heavenly Equation}

Basic generators of one-parameter subgroups of a total Lie group of point symmetries for the asymmetric heavenly system (2) has the form [2]

$$
\begin{aligned}
X_{1}= & y \partial_{y}+u \partial_{u}+v \partial_{v} \\
X_{2}= & \left(\frac{a k x}{b^{2}}-k t+a F^{\prime}(s)\right) \partial_{t}+b F^{\prime}(s) \partial_{x}+k y \partial_{y} \\
X_{d}= & \left((b t-a x) d_{y z}-v d_{y y}\right) \partial_{t}+c d_{y} \partial_{x}-b d_{z} \partial_{y}+b d_{y} \partial_{z} \\
& +\left(-\frac{1}{2} d_{y y} v^{2}+\left(\frac{1}{2} a^{2} x^{2}-a b t x+\frac{1}{2} b^{2} t^{2}\right) d_{z z}-a c t d_{z}\right) \partial_{u} \\
& +\left(-b d_{y z} v+\left(b^{2} t-a b x\right) d_{z z}-a c d_{z}\right) \partial_{v} \\
X_{f}= & f_{y} \partial_{t}+(b t-a x) f_{z} \partial_{u}+b f_{z} \partial_{v} \\
X_{g}= & g(y, z) \partial_{u} .
\end{aligned}
$$

We use a particular choice of $d=y$ for $X_{d}$ and we obtain

$$
X_{y}=c \partial_{x}+b \partial_{z}
$$

The invariants of $X_{y}$ are determined by the characteristic system as

$$
X=b x-c z, \quad Y=y, \quad T=t, \quad U=u, \quad V=v .
$$


The symmetry reduction implies the ansatz: $u=U(X, Y, T)$ and $v=V(X, Y, T)$. The total derivatives in terms of new variables become

$$
D_{x}=b D_{X}, \quad D_{y}=D_{Y}, \quad D_{z}=-c D_{X}, \quad D_{t}=D_{T}
$$

where $D$ stand for partial derivative $\partial$, i.e., $D_{x} u=\partial_{x} u=u_{x}$. Substituting this into the original system (2) and renaming $U \rightarrow u, V \rightarrow v$ and $T \rightarrow t$ we obtain new 2+1-dimensional reduced system in two component form as

$$
u_{t}=v, \quad v_{t}=\frac{v_{x}}{u_{x y}}\left(v_{y}-\frac{a c}{b}\right) \equiv Q, \quad b \neq 0 .
$$

\section{Dirac's Constraints Analysis, Symplectic and Hamiltonian Structure of Reduced System}

In general, in order to prove that 2+1-dimensional reduced system (7) is an integrable bi-Hamiltonian system, we should start with a degenerate Lagrangian and follow the same procedure as we did for asymmetric heavenly system [2]. Therefore we apply (6) to the Lagrangian given in [2] and we obtain reduced Lagrangian density for the system (7) as follows

$$
L=b\left(\frac{v^{2}}{2}-v u_{t}\right) u_{x y}-\frac{a c}{2} u_{t} u_{x} .
$$

In order to get a Hamiltonian formulation, we need to apply Dirac's constraints [4] analysis. Thus, we define the canonical momenta

$$
\Pi_{i}=\frac{\partial L}{\partial u_{t}^{i}}
$$

which satisfy the canonical Poisson brackets

$$
\left[\Pi_{i}(\xi), u^{k} \eta\right]=\delta_{i}^{k} \delta(\xi-\eta)
$$

where $\xi, \eta$ are generic names for independent variables, each of which stands for the collection of our original independent variables $x, y$. In other words, $\delta(\xi-\eta)=$ $\delta\left(x-x^{\prime}\right) \delta\left(y-y^{\prime)}\right.$ for $\xi=\{x, y\}$ and $\eta=\left\{x^{\prime}, y^{\prime}\right\}$ and using (9) we get $\pi_{u}$ and $\pi_{v}$

$$
\pi_{u}=\frac{\partial L}{\partial u_{t}}=-b v u_{x y}-\frac{a c}{2} u_{x}, \quad \pi_{v}=\frac{\partial L}{\partial v_{t}}=0
$$

that cannot be solved for velocities $u_{t}$ and $v_{t}$, and therefore the Lagrangian (8) is degenerate. Following Dirac's theory of constraints [4], we treat the definitions (11) as the second class constraints

$$
\phi_{u}=\pi_{u}+b v u_{x y}+\frac{a c}{2} u_{x}=0, \quad \phi_{v}=\pi_{v}=0
$$


and calculate the Poisson bracket of the constraints

$$
K_{i j}=\left[\phi_{i}(x, y), \phi_{j}\left(x^{\prime}, y^{\prime}\right)\right], \quad i, j=1,2
$$

where $\phi_{1}=\phi_{u}$ and $\phi_{2}=\phi_{v}$. Organizing them in the form of a matrix, we find

$$
K=\left(\begin{array}{cc}
-\left(\left(b v_{y}-a c\right) D_{x}+b v_{x} D_{y}+b v_{x y}\right) & b u_{x y} \\
-b u_{x y} & 0
\end{array}\right) .
$$

Below we show that this explicitly skew-symmetric operator is a symplectic operator in the sense of Fuchssteiner and Fokas [6]. Here the corresponding symplectic two-form is the volume integral

$$
\Omega=\int_{V} \omega \mathrm{d} x \mathrm{~d} y \mathrm{~d} z
$$

of the density

$$
\omega=\frac{1}{2} \mathrm{~d} u^{i} \wedge K_{i j} \mathrm{~d} u^{j}=b u_{x y} \mathrm{~d} u \wedge \mathrm{d} v-\frac{b}{2} v_{x} \mathrm{~d} u \wedge \mathrm{d} u_{y}-\frac{1}{2}\left(b v_{y}-a c\right) \mathrm{d} u \wedge \mathrm{d} u_{x}
$$

where $u^{1}=u$ and $u^{2}=v$. In $\omega$ under the sign of volume integral (15), we can neglect all the terms that are either total derivatives or total divergences due to suitable boundary condition on the boundary surface of the volume. For the exterior differential of this two-form we obtain

$$
\mathrm{d} \omega=b \mathrm{~d} u_{x y} \wedge \mathrm{d} u \wedge \mathrm{d} v-\frac{b}{2} \mathrm{~d} v_{x} \wedge \mathrm{d} u \wedge \mathrm{d} u_{y}-\frac{b}{2} \mathrm{~d} v_{y} \wedge \mathrm{d} u \wedge \mathrm{d} u_{x}
$$

or

$$
\mathrm{d} \omega=\frac{b}{2} D_{x}\left[\mathrm{~d} u_{y} \wedge \mathrm{d} u \wedge \mathrm{d} v\right]+\frac{b}{2} D_{y}\left[\mathrm{~d} u_{x} \wedge \mathrm{d} u \wedge \mathrm{d} v\right] \Leftrightarrow 0
$$

Here the application of total derivatives to differential forms is performed in the context of the variational complex as explained in the Olver's book, Section 5.4 [7]. In (17) $\mathrm{d} \omega$ is a total divergence, which is equivalent to zero as we have explained above, so that two-form $\Omega$ is closed and therefore it is a symplectic two-form and so $K$, defined by (14), is a symplectic operator [6]. Hence its inverse is a Hamiltonian operator

$$
J_{0}=K^{-1}=\left(\begin{array}{cc}
0 & -\frac{1}{b u_{x y}} \\
\frac{1}{b u_{x y}} & J_{0}^{22}
\end{array}\right)
$$


where

$$
J_{0}^{22}=\frac{a c}{b^{2} u_{x y}} D_{x} \frac{1}{u_{x y}}-\frac{1}{2 b}\left(\frac{v_{y}}{u_{x y}^{2}} D_{x}+D_{x} \frac{v_{y}}{u_{x y}^{2}}+\frac{v_{x}}{u_{x y}^{2}} D_{y}+D_{y} \frac{v_{x}}{u_{x y}^{2}}\right)
$$

and $D_{t}, D_{x}, D_{y}$ denote operators of total derivatives with respect to $t, x, y$, respectively. The closeness of the symplectic two-form (16) is equivalent to satisfaction of the Jacobi identity for the Hamiltonian operator $J_{0}[6]$.

The Hamiltonian density, corresponding to $J_{0}$, is defined as

$$
H_{1}=\pi_{u} u_{t}+\pi_{v} v_{t}-L
$$

which leads to

$$
H_{1}=-\frac{b}{2} v^{2} u_{x y}
$$

One can obtain the flow specified in equation (2) by applying $J_{0}$ to variational derivatives of Hamiltonian density $H_{1}$

$$
\left(\begin{array}{l}
u_{t} \\
v_{t}
\end{array}\right)=J_{0}\left(\begin{array}{c}
\delta_{u} H_{1} \\
\delta_{v} H_{1}
\end{array}\right)=\left(\begin{array}{c}
v \\
\frac{v_{x}}{u_{x y}}\left(v_{y}-\frac{a c}{b}\right)
\end{array}\right)
$$

where $\delta_{u}$ and $\delta_{v}$ are Euler-Lagrange operators [7] with respect to $u$ and $v$ applied to the Hamiltonian density $H_{1}$ (they correspond to variational derivatives of the Hamiltonian functional $\left.\int_{V} H_{1} \mathrm{~d} V\right)$.

\section{Recursion Operator and Lax Pair for Reduced System}

Lie equations for symmetries of reduced system (7) have the form

$$
\left(\begin{array}{l}
u_{\tau} \\
v \tau
\end{array}\right)=\left(\begin{array}{l}
\varphi \\
\psi
\end{array}\right) \equiv \Phi
$$

where $\varphi$ and $\psi=\varphi_{t}$ are components of the symmetry characteristic and $\tau$ is a symmetry group parameter. From the Fréchet derivative of the flow (7), we find the equation that determines its symmetries $\hat{A}(\Phi)=0$, where the operator $\hat{A}$ is

$$
\hat{A}=\left(\begin{array}{cc}
D_{t} & -1 \\
\frac{Q}{u_{x y}} D_{x} D_{y} & D_{t}-\frac{1}{u_{x y}}\left(v_{y}+\frac{a c}{b}\right) D_{x}-\frac{v_{x}}{u_{x y}} D_{y}
\end{array}\right) .
$$


The recursion operator is defined as a linear operator which maps any symmetry of a given equation again into a symmetry of the same equation. As a consequence, this operator commutes with the operator $\hat{A}$ of the symmetry condition $\hat{A}(\Phi)=0$ on solution of the latter equation and equation (7). It is obtained by a symmetry reduction from the recursion operator for the four dimensional asymmetric heavenly system corresponding to (1) that was given in [2], and reads

$$
R=\left(\begin{array}{cc}
D_{x}^{-1}\left(v_{x} D_{y}-\frac{a c}{b} D_{x}\right) & -D_{x}^{-1} u_{x y} \\
Q D_{y} & -v_{y}
\end{array}\right)
$$

where $D_{x}^{-1}$ is the inverse of $D_{x}$. The commutator of the recursion operator $R$ and the operator $\hat{A}$ of the symmetry condition has the form

$$
[R, \hat{A}]=\left(\begin{array}{cc}
-D_{x}^{-1}\left(v_{t}-Q\right)_{x} D_{y} & D_{x}^{-1}\left(u_{t}-v\right)_{x y} \\
\frac{1}{u_{x y}}\left(-c b^{2}\left(u_{t}-v\right)_{x x}+b Q\left(u_{t}-v\right)_{x y}\right. & \\
-b^{2} c\left(u_{t}-v\right)_{x x}-b v_{y}\left(v_{t}-Q\right)_{x} & \\
\left.-b v_{x}\left(v_{t}-Q\right)_{y}+a c\left(v_{t}-Q\right)_{x}\right) D_{y} & \left(v_{t}-Q\right)_{y}
\end{array}\right)
$$

and as a consequence, the operators $R$ and $\hat{A}$ form a Lax pair of the Olver-IbragimovShabat [5] type for the asymmetric heavenly system (7), so that $R$ and $\hat{A}$ commute on solutions of this system.

\section{Second Hamiltonian Structure and Hamiltonian Function}

By using theorem of Magri [8,9], one can generate the second Hamiltonian operator, by applying the recursion operator (24) to the first Hamiltonian operator $J_{1}=R J_{0}$ with the result

$$
J_{1}=R J_{0}=\left(\begin{array}{cc}
-D_{x}^{-1} & \frac{v_{y}}{u_{x y}} \\
-\frac{v_{y}}{u_{x y}} & J_{1}^{22}
\end{array}\right)
$$

where $J_{1}^{22}$ in an explicitly skew-symmetric form is defined as

$$
\begin{aligned}
J_{1}^{22}= & \frac{1}{2 b}\left(v_{y}^{2} D_{x} \frac{1}{u_{x y}^{2}}+\frac{1}{u_{x y}^{2}} D_{x} v_{y}^{2}\right)-\frac{a c}{2 b^{2}}\left(\frac{v_{y}}{u_{x y}} D_{x} \frac{1}{u_{x y}}+\frac{1}{u_{x y}} D_{x} \frac{v_{y}}{u_{x y}}\right) \\
& +\frac{1}{2 b}\left(\frac{v_{x}}{u_{x y}} D_{y} \frac{v_{y}}{u_{x y}}+\frac{v_{y}}{u_{x y}} D_{y} \frac{v_{x}}{u_{x y}}\right)-\frac{1}{2 b}\left(Q D_{y} \frac{1}{u_{x y}}+\frac{1}{u_{x y}} D_{y} Q\right) .
\end{aligned}
$$


This operator is manifestly skew-symmetric. The proof of the Jacobi identity is straightforward and lengthy. The calculation are simplified by using Olver's criterion, namely Theorem 7.8 in his book [7], formulated in terms of functional multi-vectors. Moreover, $J_{0}$ and $J_{1}$ are compatible Hamiltonian operators, that is, every linear combination $\alpha J_{0}+\beta J_{1}$ with constant coefficients $\alpha$ and $\beta$ satisfies the Jacobi identity. We again note that operator (26) could be obtained by a symmetry reduction from the second Hamiltonian structure in [2]. Thus, we obtain the second Hamiltonian form of the reduced system (7)

$$
\left(\begin{array}{l}
u_{t} \\
v_{t}
\end{array}\right)=J_{1}\left(\begin{array}{l}
\delta_{u} H_{0} \\
\delta_{v} H_{0}
\end{array}\right)
$$

with the Hamiltonian density

$$
H_{0}=b\left(c_{0}-y\right) v u_{x y}
$$

where $c_{0}$ is a constant, so that reduced system (7) is a bi-Hamiltonian system, that is, it can be written in the two Hamiltonian forms

$$
\left(\begin{array}{l}
u_{t} \\
v_{t}
\end{array}\right)=J_{0}\left(\begin{array}{c}
\delta_{u} H_{1} \\
\delta_{v} H_{1}
\end{array}\right)=J_{1}\left(\begin{array}{c}
\delta_{u} H_{0} \\
\delta_{v} H_{0}
\end{array}\right) .
$$

By repeated applications of the recursion operator to the first Hamiltonian operator $J_{0}$ according to Magri's theorem we could generate an in finite sequence of Hamiltonian operators as

$$
J_{n}=R^{n} J_{0}, \quad n=1,2,3, \ldots
$$

which proves that reduced system considered in two component form is a multiHamiltonian system in above sense.

\section{Symmetries and Integrals of Motions}

Hamiltonian operators provide a natural link between commuting symmetries in evolutionary form [7] and conserved quantities (integral of motions) that are in involution with respect to Poisson brackets. Our two-component reduced system (7) is also a member of an infinite hierarchy of symmetries. Point symmetries generators of (7) read

$$
\begin{aligned}
X_{1}= & f(x) \partial_{x} \\
X_{2}= & \left(-t g_{v}-t v h_{v}-u h_{v}-w_{v}+t h\right) \partial_{t}+e(y) \partial_{y} \\
& +\left(-t v g_{v}+t v^{2} h_{v}-u v h_{v}-v w_{v}+t g+u h+w\right) \partial_{u}+g \partial_{v}
\end{aligned}
$$


where $f(x), g(y, v), h(y, v), w(x, y, v)$ and $e(y)$ are arbitrary functions. These point symmetries are generated by integrals of motion, that is, they are variational symmetries and the integrals are given by the Hamiltonian form of Noether's theorem

$$
\left(\begin{array}{c}
\delta_{u} H \\
\delta_{v} H
\end{array}\right)=K\left(\begin{array}{l}
\hat{\eta}^{u} \\
\hat{\eta}^{v}
\end{array}\right)=\left(\begin{array}{cc}
-\left(\left(b v_{y}-a c\right) D_{x}+b v_{x} D_{y}+b v_{x y}\right) & b u_{x y} \\
-b u_{x y} & 0
\end{array}\right)\left(\begin{array}{c}
\hat{\eta}^{u} \\
\hat{\eta}^{v}
\end{array}\right) .
$$

We determine the integral of motion $H$ (for a variational symmetry), corresponding to known symmetry characteristics $\hat{\eta}^{u}, \hat{\eta}^{v}$ via the relation (33). For the first symmetry $X_{1}$, the corresponding symmetry characteristic is: $\hat{\eta}^{u}=-u_{x} f(x)$, $\hat{\eta}^{v}=-v_{x} f(x)$ and we obtain

$$
H^{1}=\left(b v u_{x} u_{x y}+\frac{a c}{2} u_{x}^{2}\right) f(x)
$$

which is the conserved density corresponding to the first point symmetry $X_{1}$. For the second point symmetry $X_{2}$, we can find an integral of motion only for a special choice of arbitrary functions. For example, if we choose $h=0, w=0, g=b$ and $e=1$ we obtain

$$
X_{2}=\partial_{y}+b t \partial_{u}+b \partial_{v}, \quad \hat{\eta}^{u}=b t-u_{y}, \quad \hat{\eta}^{v}=b-v_{y} .
$$

Using (35) into (33) we get

$$
H^{2}=b v u_{x y}\left(u_{y}-b t\right)+\frac{1}{2}\left(b^{2}-a c\right) u u_{x y} .
$$

It may be possible to find different integrals of motion for different choices of arbitrary functions.

\section{Conclusion}

We have shown that a certain symmetry reduction of the 3+1-dimensional asymmetric heavenly equation, taken in a two-component form yields a two component $2+1$-dimensional multi-Hamiltonian integrable system. For this system, we have presented explicitly two Hamiltonian operators, a recursion operator for symmetries, a complete set of point symmetries and corresponding integrals of the motion. The first impression of the major part of this work could be that it is an easy and even trivial task to obtain a three-dimensional multi-Hamiltonian system by a symmetry reduction of the original four-dimensional asymmetric heavenly system. All main objects $J_{0}, J_{1}, K, \hat{A}, R, H_{0}, H_{1}$ and $L$ could be obtained by the symmetry 
reduction. However, a slight change in a symmetry chosen for the reduction, ruins all these properties and creates a difficulty in discovering bi-Hamiltonian structure of the reduced system. If we choose more general symmetries for the reduction, for example from the optimal system of one-dimensional subalgebras from [2], then we shall be unable to discover even a single Hamiltonian structure of reduced systems. The problem of conservation of multi-Hamiltonian structure under symmetry reductions seems to be an important and interesting subject for a future research.

\section{Acknowledgements}

The authors would like to thank to Mikhail Sheftel for his useful advices.

\section{References}

[1] Sheftel M. and Malykh A., On Classification of Second-Order PDEs Possessing Partner Symmetries, J. Phys. A: Math. \& Theor. 42 (2009) 395202 (20pp).

[2] Yazici D., Bi-Hamiltonian Struture of an Asymmetric Heavenly Equation, J. Phys. A: Math. \& Theor 44 (2011) 505203 (10pp).

[3] Plebanski J., Some Solution of Complex Einstein Equations, J. Math. Phys. 16 (1975) 2395-2402.

[4] Dirac PAM, Lectures on Quantum Mechanics, Yeshiva University Press, New York 1964.

[5] Ibragimov N. and Shabat B., Evolutionary Equations with Nontrivial LieBacklund Algebra, Funct. Anal. Appl. 14 (1980) 19-28.

[6] Fuchssteiner B. and Fokas A., Symplectic Structures, Their Backlund Transformation and Hereditary Symmetries, Physica D 4 (1981) 47-66.

[7] Olver P., Applications of Lie Groups to Differential Equations, Springer, New York 1986.

[8] Magri F., A Simple Model of the Integrable Hamiltonian Equation, J. Math. Phys. 19 (1978) 1156-1162.

[9] Magri F., Nonlinear Evolution Equations and Dynamical Systems, In: Lecture Notes in Physics vol. 120, M. Boiti, F. Pempinelli and G. Soliani (Eds), Springer, New York 1980, pp 233-263. 
Devrim Yazıc1

Department of Physics

Yıldız Technical University

Istanbul 34220, TURKEY

E-mail address: yazici@yildiz.edu.tr

\section{Hakan Sert}

Department of Physics

Yıldız Technical University

Istanbul 34220, TURKEY

E-mail address: hakansert13@hotmail.com 